\title{
Optical Characterization of Mineral Dust from the EAIIST Project with Digital Holography
}

\author{
Claudia Ravasio,* Llorenç Cremonesi, Claudio Artoni, Barbara Delmonte, Valter Maggi, \\ and Marco A. C. Potenza
}

Cite This: ACS Earth Space Chem. 2021, 5, 2855-2864

Read Online

\section{ACCESS | Lلll Metrics \& More | 回 Article Recommendations | st Supporting Information}

ABSTRACT: We describe an optical approach based on Digital Holography for single-particle characterization of mineral dust and micrometric particles, focusing on the analysis of airborne particles in meltwater from Antarctic ice cores. We record the holograms formed by the superposition of the transilluminating reference beam and the waves scattered by single particles. Taking a cue from recent approaches in the field and holography methods, we process the holograms to recover both optical and morphological properties of single dust grains. As a considerable advantage over traditional light-scattering-based methods, holograms give the extinction cross
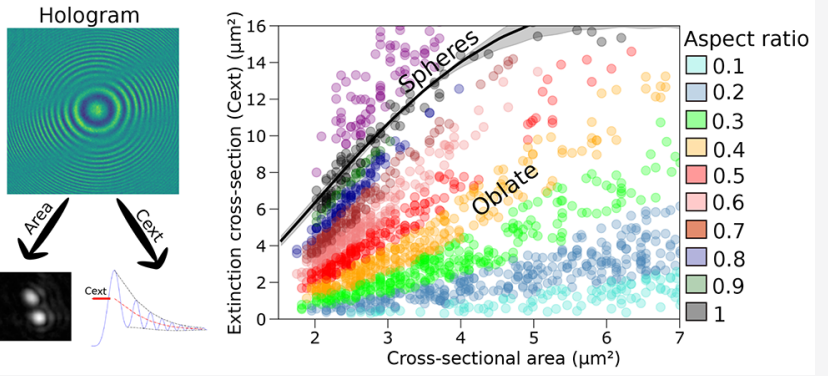
section of each particle and, by numerically reconstructing the wavefront propagation, an unambiguous image of each particle whereby we derive its cross-sectional shape and size. Measurements have been carried out on samples collected from the recent EAIIST (East Antarctic International Ice Sheet Traverse) project, some of which show evidence of volcanic events. The vast majority of the detected particles show significant deviations from the isometric shape, as confirmed by both image reconstruction and extinction cross section analysis. By our analysis, we observe that experimental data have an extinction cross section up to 3 times lower than that of spherical particles with the same volume. Therefore, these deviations have an appreciable impact on the aerosol contribution to radiative forcing: retrieving particle shape may improve the modeling of the radiative properties of mineral dust and reduce the associated uncertainties.

KEYWORDS: Digital Holography, Mineral dust, Aerosols, Ice core, Optical properties

\section{INTRODUCTION}

Despite the well-recognized importance of past changes in mineral dust aerosol distribution and content for the estimates of climate sensitivity, ${ }^{1,2}$ the role of dust in climate change still represents an important source of uncertainty in climate models. Although extensive research has been carried out on this topic, significant uncertainties still affect current estimations. The effect of aerosols on the atmospheric system has been found to be comparable to other sources, including greenhouse gases such as $\mathrm{CO}_{2}$ and $\mathrm{CH}_{4} \cdot{ }^{3-5}$ This contribution is tightly related to their optical properties, which dictate their effectiveness in scattering and absorbing solar and terrestrial radiation, ${ }^{6}$ as well as the morphological and chemical properties that determine their reactivity in the atmosphere and the ability to induce the nucleation of water droplets and ice crystals. ${ }^{1,7,8}$

Among aerosols, mineral dust is one of the most significant contributors to the dry mass particle (long-range) load in the troposphere. ${ }^{9,10}$ It consists of insoluble, micron-sized minerals from both natural and anthropogenic sources entrained in the atmosphere by aeolian processes, part of which is deposited on the cryosphere and in water. ${ }^{11-13}$ Mineral dust exhibits the largest size distribution variability among aerosols: while dispersion close to the major dust source regions includes all size ranges, up to coarse silt and fine sand, only clays and very fine silt are involved in long-range transport. In central East Antarctica, where dust is transported from Southern Hemisphere continents and the mean transit time is on the order of 3-4 weeks, ${ }^{14}$ the modal value of the volume (mass) dust size distribution is around $2 \mu \mathrm{m}$, with small variations mainly related to the altitude and type of transport. ${ }^{15}$

Besides grain size and complex refractive index, singleparticle light scattering and absorption depend on many parameters: for example, an increasing amount of literature is pointing out the importance of particle shape and morphology (aggregation state, coating, surface roughness) when approaching an inversion of the scattering data or modeling their impact on energy balance. ${ }^{16-20}$ Moreover, deviations from ideal spheres have an appreciable impact on the radiative forcing

Received: June 23, 2021

Revised: August 2, 2021

Accepted: August 5, 2021

Published: September 1, 2021 
from aerosols. ${ }^{21}$ Overall, these results support the conclusion that one of the main reasons for the uncertainties affecting the impact of aerosols stems from the spherical shape approximation in the state-of-the-art numerical climate models, ${ }^{22,23}$ and that they would benefit from more accurate modeling of the aerosol components.

Digital holography $(\mathrm{DH})$ is a technique that has been widely used in the fields of optics. It is well suited for the investigation of microcomponents, for dynamic three-dimensional particle field imaging, and tracking systems. It offers a noncontact approach, preserving the samples, and it can cover a wide range of sizes. For these reasons, it has many applications that include studying contaminants in water, ${ }^{24}$ quantifying unconstrained bacterial mobility, ${ }^{25}$ studying protein aggregates, ${ }^{26}$ or characterizing flowing aerosol particles in air. ${ }^{27}$

In this work, we propose for the first time the application of $\mathrm{DH}$ to study meltwater samples from polar snow, and we designed a dedicated apparatus and flow system for this purpose. DH allows one to obtain a direct measurement of (1) the extinction cross section and (2) an image of their silhouette when crossing so that the shape of the particles can be inferred. Particle-by-particle optical measurements contribute significantly to an all-round characterization of mineral dust by giving direct access to their optical properties, especially when many parameters are measured simultaneously and independently. In this work, particular attention was given to the shape of the particles which consistently exhibit a considerable nonsphericity. Data were also compared with results from particle volume measurements obtained by a Coulter Counter.

\section{METHODS: DIGITAL HOLOGRAPHY}

The experimental setup is outlined in Figure 1. Light from a visible laser source $(\mathrm{He}-\mathrm{Ne}$ laser $5 \mathrm{~mW}, 633 \mathrm{~nm}$, about 0.8

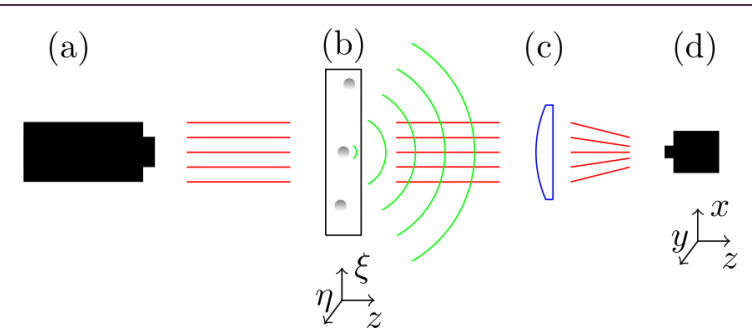

Figure 1. Diagram of in-line Digital Holography. A laser beam (a) is focused and spatially filtered by a $30 \mu \mathrm{m}$ pinhole (not shown); the collimated beam illuminates a thin cell $(b)$ through which particles (gray spheres) are flown. A 20× microscope objective (c) is located in the far-field of the sample, and a sensor (d) records the holographic pattern resulting from the interference of the faint scattered (green lines) and transmitted waves (red lines).

$\mathrm{mm}$ in width) is focused with a $20 \times$ objective, spatially filtered by a $\sim 30-\mu$ m-diameter pinhole, and collimated with a $4 \times$ objective. The pinhole filters the beam improving its spatial coherence and enhancing the quality of the holograms. The collimated beam impinges onto a quartz flow-cell centered on the scattering volume $(5 \times 0.2 \mathrm{~mm}$ cross section $)$. A $20 \times$ microscope objective is located in the far-field to reach the desired spatial resolution. Finally, the beam reaches a CMOS detector (Ueye-5240CP-M-GL IDS Camera), illuminating its whole pixel array $(1280 \times 1024,5.3 \mu \mathrm{m}$ pixel size $)$. The custom, table-top instrument is kept inside a laminar flow hood to prevent contamination of the samples.

The samples are melted and transferred into a borosilicate glass container that serves as a reservoir; a steady stream of $\sim 2$ $\mathrm{mL} \min ^{-1}$ is delivered to the illuminated region using a peristaltic pump. Broadly speaking, any small particle intercepting the laser beam scatters an almost spherical wave, which in turn interferes with the trans-illuminating field, similarly to in-line Gabor's holography. ${ }^{28}$ The resulting selfreference interference pattern conveys information on both the amplitude and the phase of the scattered field. An example of the output of the instrument is shown in the SI (Figures S2 and S3).

The design of the instrument is simple and compact; its case is approximately $80 \times 20 \times 20 \mathrm{~cm}^{3}$. Among the advantages of $\mathrm{DH}$ over other optical techniques is that the accurate alignment of its optical components is not critical; hence, the setup is hardly affected by issues related to misalignment. Moreover, as it does not require time-consuming sample preparations, it is suited for in-line applications. ${ }^{29,30}$ Other factors contribute to the robustness of this method. First, it is calibration-free, only needing a fine-tuning of the magnification. Second, it works well under strong dilution conditions (typically limiting static light scattering); hence, it is particularly suited for polar ice core and snow samples. Low particle concentration in the samples prevents the overlap of waves from multiple dust particles. The main criticalities, such as the occurrence of multiple events, can be handled with the custom-made software. For example, it is important to distinguish an aggregate from several separate particles, since the optical properties of these two morphologies are different even if the total mass is the same. We also note that low refractive index impurities such as bacteria give a low-intensity contrast in the images and do not pose a problem during measurements: repeating measurements after several hours gave consistent results. The working principle of the instrument makes it suitable for continuous flow analysis systems typically used in ice core characterization; this can be further eased by optimizing the camera field of view and the thickness of the flow cell.

Optical Properties. The interaction of a wave with a wavelength-scale particle causes a small attenuation of the original beam: the particle scatters and possibly absorbs part of the incoming radiation. Extinction is the overall effect that describes this power reduction and is quantified by the particle's extinction cross section $\left(C_{\text {ext }}\right)$ (Table 1$) .{ }^{31-33}$ Since the hologram is a consequence of the interference between the scattered and transmitted fields, the holographic pattern also contains information about the $C_{\text {ext }}$ of the particle. As a first

Table 1. List of Symbols and Abbreviations

\begin{tabular}{|c|c|}
\hline description & symbol \\
\hline Digital Holography & $\mathrm{DH}$ \\
\hline Extinction cross section & $C_{\text {ext }}$ \\
\hline Cross-sectional area, orthogonal to the optical axis & $c s a$ \\
\hline Aspect ratio, the ratio between the major and minor axes of the csa & ar \\
\hline Thickness (along the optical axis) to diameter ratio & $t d r$ \\
\hline Coordinates on the hologram plane & $(x, y)$ \\
\hline Coordinates on the objects plane & $(\xi, \eta)$ \\
\hline Wavenumber & $k=\frac{2 \pi}{\lambda}$ \\
\hline Complex scattering amplitude at zero angle & $S(0)$ \\
\hline
\end{tabular}



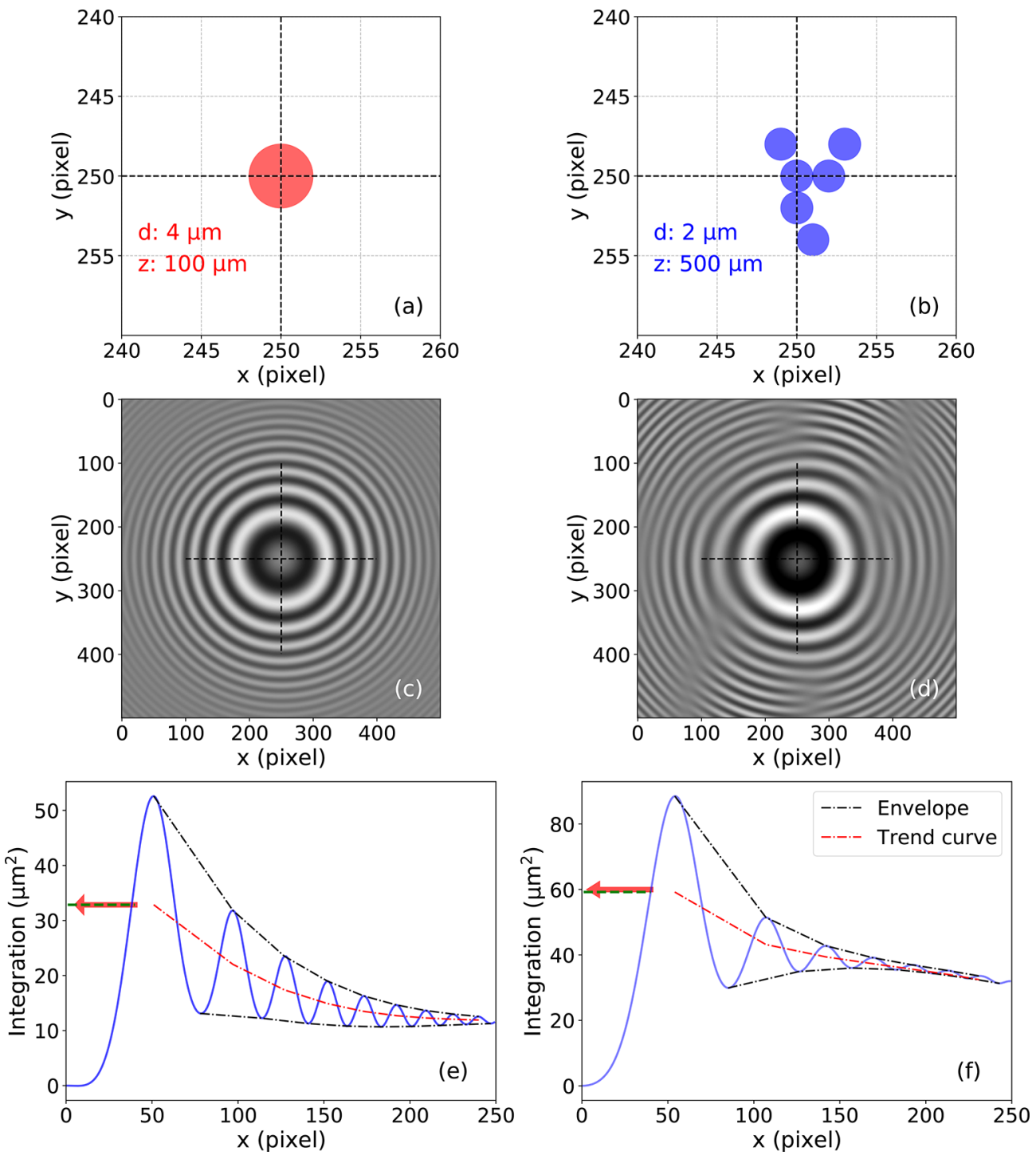

Figure 2. Examples of a general method to extract the extinction cross section from a hologram in two simulated cases. (a, b) Different particle morphologies: sphere and aggregate. (c, d) Intensity plots of the corresponding holograms; the centered dashed lines correspond to the position of the dashed lines in the top images. (e, f) Integration of the corresponding holographic pattern by transition square matrix from the first maximum to the edges. Following integration, the end point of the trend curve (red dashed line) corresponding to the first maximum gives the $C_{\text {ext }}$ (red arrow), in good agreement with the expected value (green dashed line).

approximation, the interference pattern can be described by a sinusoidal function with a phase shift $\phi$ and a decrease in amplitude with respect to the incident wave caused by the presence of the particle. The zero-angle value of the complex scattering amplitude is denoted by $S(0)$. In this framework, we can directly obtain the value of $S(0)$ and the phase shift $\phi$ by approximating the hologram pattern generated by a spherical object as ${ }^{31}$

$$
I(x, y)=\frac{S(0)}{k z} \cos \left(k \frac{\left(x-x_{0}\right)^{2}+\left(y-y_{0}\right)^{2}}{2 z}+\phi\right) f(\theta)
$$

where $f(\theta)$ is the form factor, a characteristic function of the interference pattern accounting for the intensity damping as the scattering angle increases, $\left(x_{0}, y_{0}\right)$ denotes the position of the scatterer on the plane, $z$ is the position along the optical axis, and $k$ is the wavenumber of the incoming radiation. Finally, by restricting ourselves to a lossless medium, the
Optical Theorem states that the total scattering cross section is $C_{\text {ext }}=\left(4 \pi / k^{2}\right) \operatorname{Re} S(0),{ }^{31}$ where the real part of $S(0)$ is defined as $|S(0)| \cos \left(\frac{\pi}{2}-\phi\right)$. A list of symbols and abbreviations is given in Table 1.

In Berg et al., ${ }^{34}$ the authors state that measuring $C_{\text {ext }}$ is equivalent to the difference of the net response of a sensor looking into the light source with and without an arbitrary particle disturbing the incident wave. This operation is the same as integrating the contrast hologram, namely, the hologram pattern without the background. This procedure is sketched in Figure 2. Two different cases are considered (panels a and b): a single sphere and an aggregate of several particles (the corresponding dimensions and axial positions are indicated in the plot). The simulated particles produce two hologram patterns (Figure $2 \mathrm{c}$ and $\mathrm{d}$ ) that vary significantly according to the scatterer morphology. By integrating the pattern from its center to the edges, the value of $C_{\text {ext }}$ can be obtained by measuring the envelopes of the integration curve 
(a)

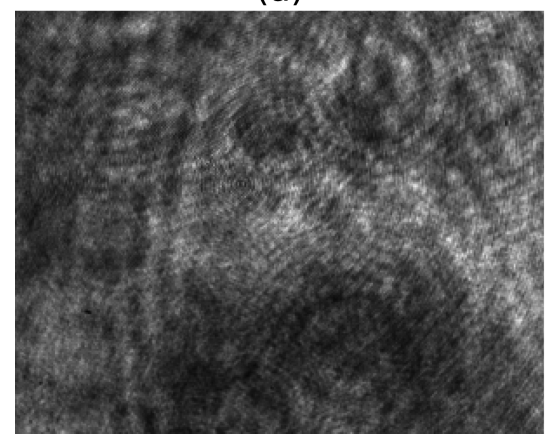

(b)

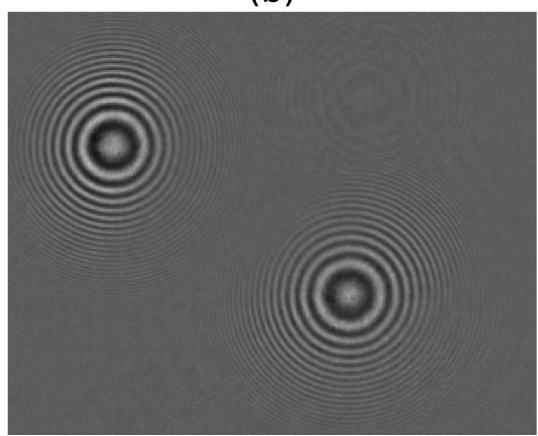

Figure 3. From the raw image (a) we subtract the background image and produce a normalized frame where the holograms are clearly distinguishable (b).

(a)

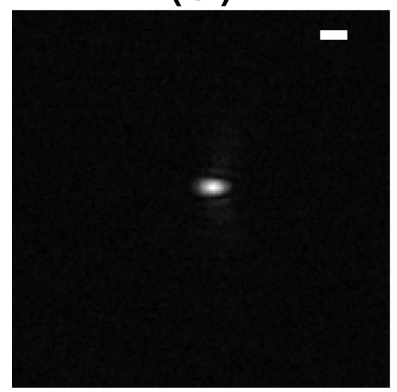

(d)

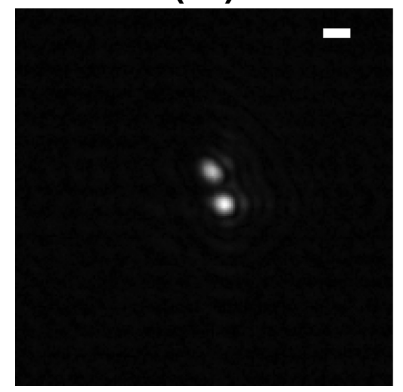

(b)

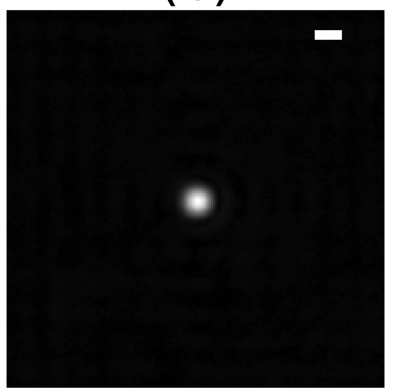

(e)

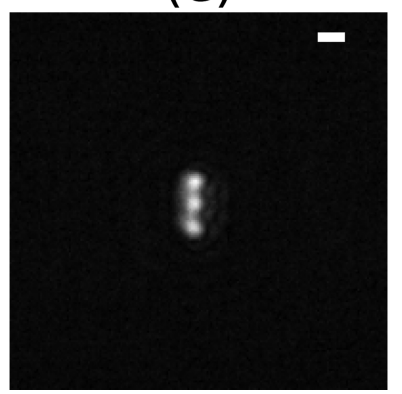

(c)

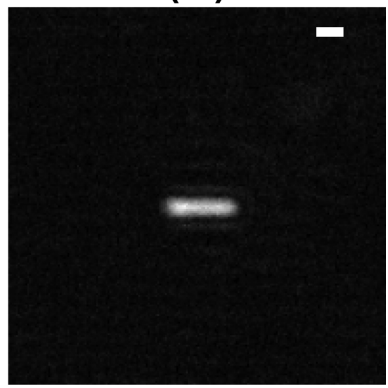

(f)

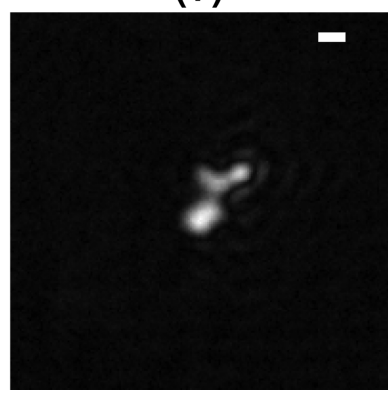

Figure 4. Examples of some reconstructed images from some contrast holograms. A wide variety of shapes (cross-sectional areas) is observed, although spheroidal and needle-like dust particles are most common (a,b,c). The white bar on the top-right corner of each image is set to $2 \mu \mathrm{m}$.

(black, dashed line in Figure 2e and $\mathrm{f}$ ), which define the average trend (red, dashed line). An estimate for $C_{\text {ext }}$ is obtained by extrapolating the end point of the trend curve, denoted by the horizontal red arrow in the plots and corresponding to the scattering angle $\theta=0$. Using the opensource package HoloPy (https://github.com/manoharan-lab/ holopy), we calculate the expected $C_{\text {ext }}$ (green dashed line) by computing the scattered fields using Mie theory (e) and a Tmatrix-based solution of scattering that accounts for multiple scattering between spheres (f). Results show that this method works well both for symmetrical objects and aggregates. ${ }^{35}$

Image Analysis and Object Reconstruction. Images are recorded continuously in real time $(1280 \times 1024$, grayscale, 8 bit scale), while the reconstruction is in postprocess. An algorithm sets a threshold on the variance of the recorded images and detects particles inside the field of view of the instrument. Since the amplitude of the scattered wave approximately decays as $r^{-1}$, the contribution of each scatterer is confined to a limited region of the image. Hereinafter, we refer to these regions of interest as holographic features: these need to be isolated from each experimental raw image and separately analyzed. As a preprocessing step, we compute a pixel-wise median to subtract the incident beam and suppress spurious contributions such as any laser intensity fluctuations. Each pixel of the resulting image is the median of the corresponding pixel in all images. The resulting background is subtracted from the raw images to obtain the contrast holograms, which are then normalized (Figure 3).

The second step is feature localization. In each image, the center of the particle can be identified by performing a cross correlation between the normalized hologram and a spherical function damping in intensity (as eq 1). In this way, the hologram is transformed by the deconvolution into sharply resolved peaks, whose centers correspond to the position of the particle on the observation plane. More than one distinguishable particle may appear in the same image. Given the importance of accurately finding the center of the holograms on the $x y$ plane, we double-check it by implementing a Houghbased holographic feature detection algorithm. ${ }^{36}$ Once the positions of the scatterers are retrieved, the original image is 
cropped to discard the portions of the image that do not include any fringes to reduce the computational cost. If an image includes many holographic features, it is divided into subimages. Incomplete data is discarded: a threshold is set to disregard any hologram too close to the edges so that only image portions between $400 \times 400$ and $1000 \times 1000$ pixels are collected. Rejecting some events causes the apparent concentration to be slightly lower than the actual absolute concentration, an effect that can be compensated for.

As mentioned in the Supporting Information (SI), the position of the scatterer on the $(\xi, \eta)$ plane can be deducted directly from the hologram (Figure S4). Conversely, the position of the scatterer along the optical axis $(z)$ must be reconstructed numerically. To do so, we propagate the wavefront recovered by the hologram ( $\left.E_{\text {scat,hp }}\right)$ to another position along the optical axis $\left(E_{\text {scat }}\right)^{37,38}$ by the FresnelKirchhoff diffraction formula:

$$
E_{\text {scat }}(\xi, \eta, z)=\frac{i}{\lambda \rho} \iint E_{\text {scat,hp }}(x, y) \mathrm{e}^{i k \rho} \mathrm{d} \xi \mathrm{d} \eta
$$

where $\rho=\sqrt{(x-\xi)^{2}+(y-\eta)^{2}+z^{2}}, \lambda$ is the wavelength, $k$ is the wavenumber, and $(x, y)$ are the coordinates on the hologram plane (on the CMOS sensor), while $(\xi, \eta, z)$ is an arbitrary point related to the field on the observation plane (close to the cell).

Finally, having determined the position of the particle along all three axes (SI), we are able to reconstruct the particle on the object plane by a specific algorithm and retrieve their silhouettes, i.e., the cross-sectional area (csa), as follows. The images are processed with an edge detection algorithm that defines a contour enclosing each particle, designed to ignore regions affected by noise that cause the contour to be too small. If the algorithm identifies more than one particle, as in Figure $4 d$, data are rejected to avoid artifacts that would lead to an overestimation of $C_{\text {ext }}$. We impose a threshold to transform the gray-scale images into a binary images, where 1 is the particles and 0 is the background. By multiplying the area by the square pixel metric size, we obtain the csa of the particles. We then compute the major and minor axes of a (rotated) bounding rectangle around each particle to obtain their crosssectional height and width: the ratio between the major and minor axes gives the dimensionless aspect ratio (ar). Finally, in the special case where the two axes are equal $(a r \simeq 1)$ we can uniquely define the dimensionless thickness (longitudinal axis along the optical axis) to diameter ratio $(t d r)$. From the reconstructed images and the slopes of the major and minor axes, we can observe the orientation of the particles in the field of view, and we can state that almost all $(\sim 90 \%)$ are oriented along the flow direction, as expected. ${ }^{39}$

At the current stage, it is hard to precisely determine the particle throughput of the instrument. However, we can identify and discuss some limiting factors: (1) technological limits and (2) methodological limits. The former are essentially due to the limited field of view of the camera, which currently is about $3 \%$ of the effective surface of the flow cell, since we magnify the field with an objective lens. This does not introduce any instrumental biases and can be optimized with a larger sensor or a smaller flow cell (a wider field of view). The methodological limits are harder to compensate for and are mainly due to the signal-to-noise ratio: only particles with a $C_{\text {ext }} \gtrsim 0.3 \mu \mathrm{m}^{2}$ can be analyzed successfully. Below this threshold, we cannot distinguish a very faint hologram pattern from an "empty" image (with no particles in the field of view). We stress that this limit does not depend on the power of the laser, since the holograms are normalized.

\section{RESULTS AND DISCUSSION}

After validating the apparatus with polystyrene microparticles, as described in the SI (Figure S1), we measured meltwater samples from snowpits collected along the East Antarctic International Ice Sheet Traverse (EAIIST project) across the East Antarctic plateau, where dust concentration is very low $\left(\sim 10^{2}-10^{3} \mathrm{ppbv}\right)$. Samples (from the surface down to a depth of $175 \mathrm{~cm}$ depth) have been selected from five sites, which are AGO5 (coordinate), megadunes on the accumulation (MA) and on the erosion side (ME and ME2), and Wind Crust (WC, coordinate).

We obtained the $C_{\text {ext }}, c s a$, and ar as defined in the previous section. We observe a wide variety of particle shapes, as revealed by their silhouettes. Isometric and needle-like dust particles are most common (Figure $4 a, b$, and c), although we found some occurrences of aggregates of adhering particles (see Figure $4 \mathrm{e}$ and $\mathrm{f}$ ). Generally, we observe a range of sizes from 1 to $20 \mu \mathrm{m}^{2}$ and a broad $C_{\text {ext }}$ distribution (Figure 5).

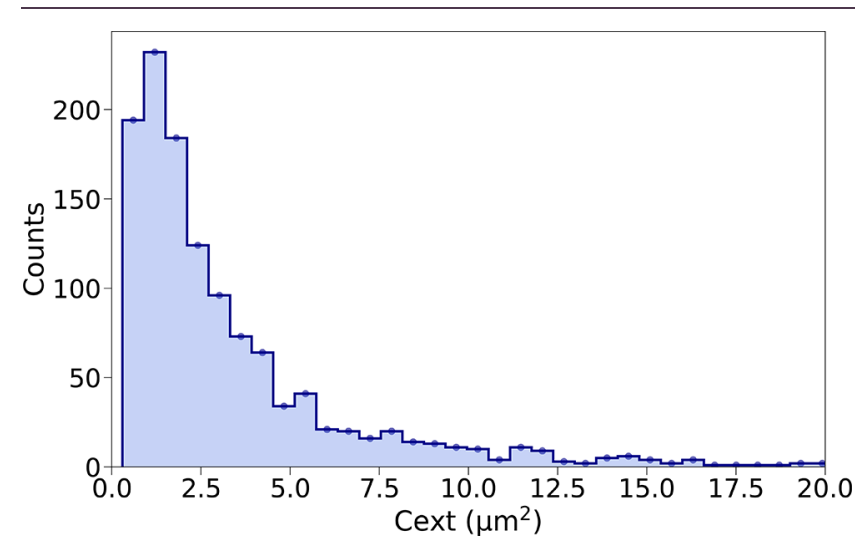

Figure 5. $C_{\text {ext }}$ distribution from AGO5 (depth $69 \mathrm{~cm}$ ). The wide distribution covers a range of values from 1 to $\sim 20 \mu \mathrm{m}^{2}$. Larger sizes can be ascribed to aggregates and are discarded.

Coulter Counter results reveal many mineral dust particles below the micrometric size range $(0.5-1 \mu \mathrm{m}$ approximately) that is inaccessible to our apparatus. However, as pointed out in the Methods, with this study we focus on the larger size range to better estimate the effects of shape.

The first thing to note is that no significant correlation between $C_{\text {ext }}$ data and csa was found in any of the samples, which would instead be expected for sphere-like particles (see for example Figure 8 below). In the histograms shown in Figure 6, the area distribution of particles with isometric csa, obtained by the holographic reconstruction algorithm, is compared to the cross-sectional area distribution by inverting $C_{\text {ext }}$ with spherical particles. The measured radius is $\sim 1.5$ times that of the $C_{\text {ext }}$-equivalent radius. The spherical approximation distorts the distribution considerably, hence the importance of measuring two parameters simultaneously and independently. Similarly, trying to obtain the $C_{\text {ext }}$ distribution from a volumeequivalent spherical model gives rise to discrepancies. In addition to optical extinction, information is needed about single particle morphology, such as particle shape and orientation. For example, flat particles cause a lower extinction of the incoming light compared to spheres for any given $c s a$; it 


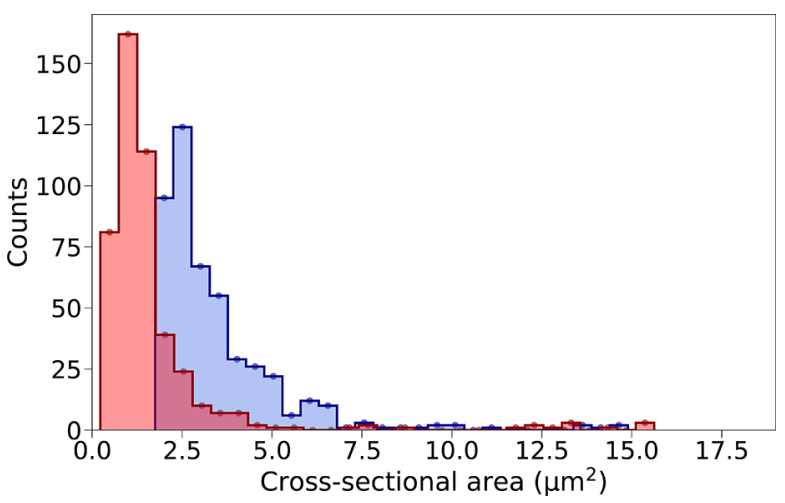

Figure 6. (Blue histogram) csa (area) distribution for ME (depth 99 $\mathrm{cm}$ ) for isometric particles obtained from the reconstruction algorithm. (Red histogram) Area distribution for the same sample obtained from the $C_{\text {ext }}$ distribution, interpreting particles as spheres.

is crucial to take into account this feature in order to compare optical data to measurements of the volume of the particles. ${ }^{39}$

Different sites exhibit considerably different shape distributions, as shown in Figure 7, where we report the ar

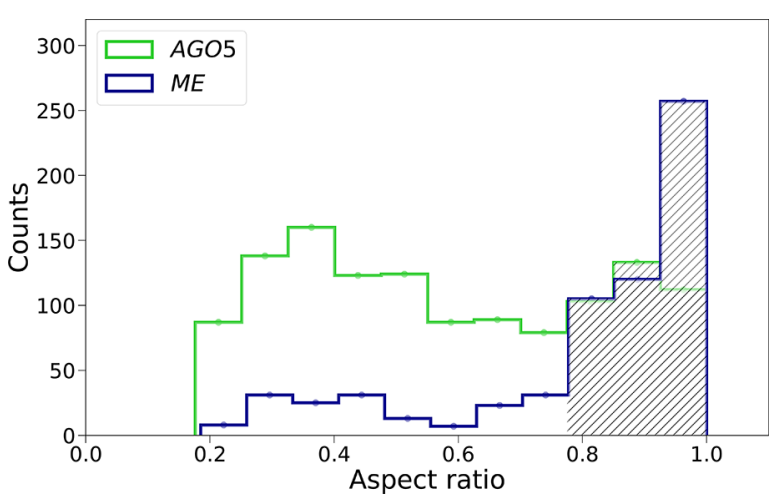

Figure 7. ar distribution from AGO5 (depth $69 \mathrm{~cm}$ ) and from ME (depth $99 \mathrm{~cm}$ ). The dashed columns are the subset of particles whose aspect ratio approaches 1 .

distributions of two samples from ME and AGO5, respectively. For ME at $99 \mathrm{~cm}$ depth (blue histogram in Figure 7), a peak at around $a r=1$ is clearly visible, a marker of isometric shapes, while the distribution of AGO5 at $69 \mathrm{~cm}$ (green histogram) shows significant shape polydispersity. In order to compare the spherical model to our data, we consider the subset of particles whose ar approaches 1 (dashed columns in Figure 7). We plot $C_{\text {ext }}$ vs area in Figure 8. To compare to the expected curves for spherical particles, the $C_{\text {ext }}$ of spherical particles were calculated with the open-source package HoloPy. The expected refractive index of the particles in our samples is between 1.51 and 1.55 ( $\sim 1.14-1.16$ relative to water), which is coherent with the expected Holocene dust composition observed at Dome C, mainly composed of plagioclase, feldspars, quartz, and phyllosilicates. ${ }^{40}$ We chose 1.15 as an average value; the refractive index did not prove to be a critical parameter in this range, since variations in $C_{\text {ext }}$ due to particles with different refractive indexes are negligible compared to the contribution of shape, specifically, to the distribution of $t d r$ in the population of particles. In addition, we were able to assess that in the samples there are almost no particles characterized by high absorption such as combustion byproducts, which are marked by an exceptionally high $C_{\text {ext }}$ for any given size. The

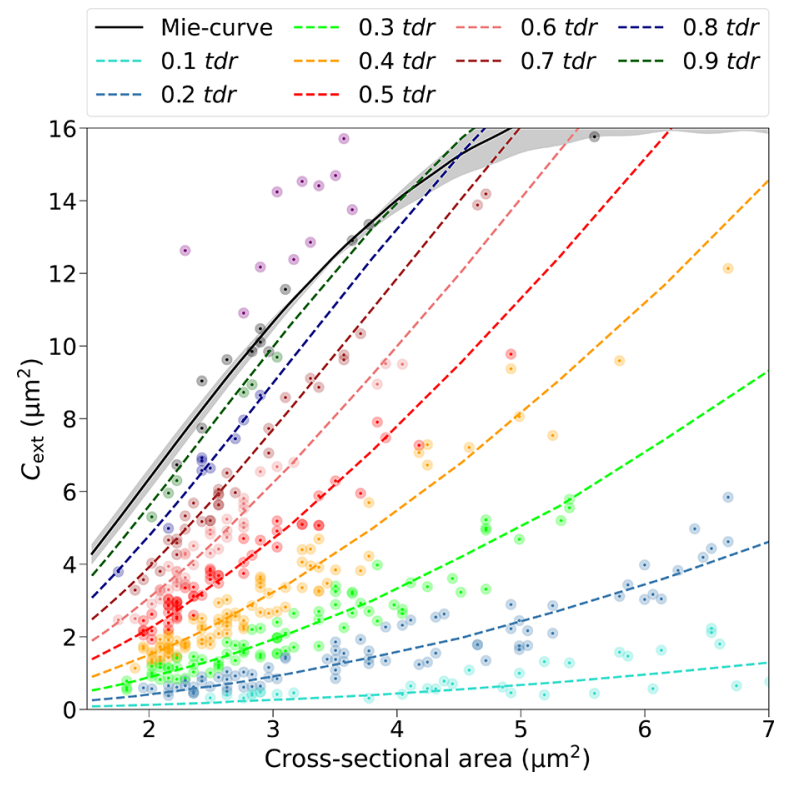

Figure 8. Scatterplot of the extinction cross section vs cross-sectional area for an ME sample (depth $99 \mathrm{~cm}$ ). The solid black line is the Mie curve for spheres (1.15). The colored lines refer to the extinction cross section of oblate ellipsoids ( 1.15 refractive index) with a ranging thickness/diameter ( $t d r$ ) from 0.1 (turquoise dashed line) to 0.9 (dark-green dashed line). The data points, whose color indicates the $t d r$ as in the corresponding dashed lines, are a subset of particles whose aspect ratio on the $\xi \eta$ plane approaches 1 .

solid black line in Figure 8 is calculated with the Lorentz-Mie model for spheres with a diameter ranging homogeneously from 1.4 to $2.8 \mu \mathrm{m}$ and a relative refractive index of 1.15 . Particles that are nearly isometric fall close to this line. We include an uncertainty band in gray obtained for Mie calculations with a refractive index ranging between 1.14 and 1.16. We note that data appear appreciably spread out at any given size, and $C_{\text {ext }}$ spans over an extended range ( $\sim 1$ order of magnitude). Such variability is attributable to the nonisometric shape of the particles. Specifically, our samples are dominated by flat (oblate) particles, whose $C_{\text {ext }}$ is consistently lower than it is for spheres, or any equivalent isometric shape.

The extinction and scattering cross sections of nonspherical particles can be calculated with the Amsterdam Discrete Dipole Approximation (ADDA) code. $^{41}$ We used ADDA to determine the $C_{\text {ext }}$ of oblate ellipsoids ( 1.15 relative refractive index) with a ranging thickness over diameter ratio (from $t d r=0.1$, turquoise dashed line, to 0.9, dark green dashed line in Figure 8). Some data points in the top-left corner of the plot lay well above the Mie curves at very high values of extinction. The former are reported as purple points in Figure 8 (ME, depth 99 $\mathrm{cm})$ and are about 5-6\% of the total counts, and are essentially due to particles with a non-negligible absorption cross section. Some of these events arise when many particles located at different $z$ coordinates occupy the scattering volume and only one of them is tracked by the reconstruction algorithm. While the other particles still contribute to the total extinction, the contrast of their holograms is too low and the estimate of the particle size is altered. However, these events are statistically negligible due to the low concentration of the samples.

By comparing the $C_{\text {ext }}$ of oblate ellipsoids with our data, we can obtain the distributions of $t d r$ from the subset of particles whose ar approaches 1 (Figure 9). In doing so, we can also 


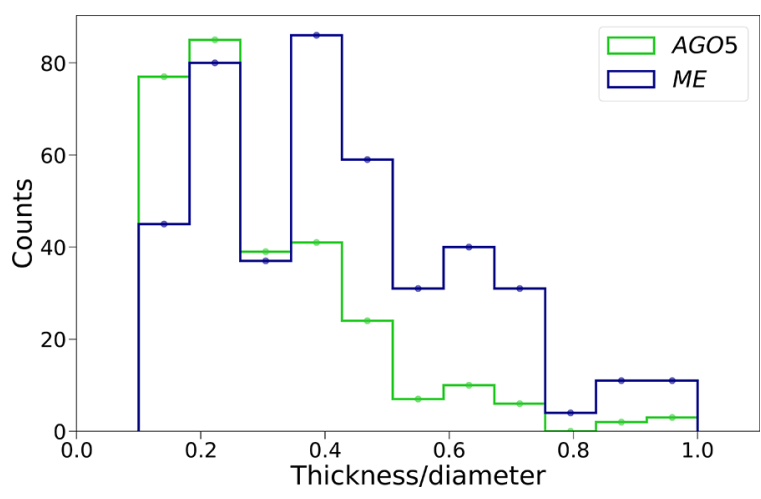

Figure 9. $t d r$ distributions obtained by comparing the simulated $C_{\text {ext }}$ of oblate ellipsoids (with isometric cross-sectional area) with our data from AGO5 (depth $69 \mathrm{~cm}$ ), and from ME (depth $99 \mathrm{~cm}$ ).

implicitly use the fact that the majority of particles orient themselves orthogonally to the optical axis. The shape of the final distribution does not have a peak around 1 (a trait of isometric particles) but extends over a wide range with a prevalence of 0.2/0.4 $t d r$ (plate-shaped particles). Moreover, the distributions in Figure 9 exhibit a similar trend, showing a comparable presence of plate-shaped particles. Once obtained the thickness of the particles, we can univocally determine the volume. At any given volume, the observed (oblate) particles with $t d r=0.1$ have an extinction $1 / 3$ times that of the equivalent spherical particles. As the $t d r$ value increases, the oblate particles have a $C_{\text {ext }}$ that tends to that of equivalent spheres $(\sim 1.1$ times for $t d r=0.6$ and 0.8 times for $t d r=0.8)$, as expected.

We summarize our results in Figure 10, where we include all the samples, selected so as to cover a variety of depths for each of the five sites. We show the parameters described above for each sample: the $C_{\text {ext }}$ (Figure 10a), the csa (Figure 10b), and the $t d r$ (Figure 10c). The data points in the plot are the medians of the respective optical and geometrical distributions. Broad distributions characterize both the area and the $C_{\text {ext }}$ but appear to be homogeneous among the different sites. We obtained the $t d r$ distributions for all the samples that show a significant number of particles whose ar approaches 1 . The plot underlines the prevalence of a principal $t d r$ range between 0.15 and 0.4 . The gray regions in Figure 10 highlight samples close to the surface that are exposed to contamination and are therefore excluded from the following analysis.

Finally, in Figure 11, we show the average concentration of the five sites compared with Coulter Counter results, which deserve some further considerations. Some volcanic events have been identified in several sites at different depths (i.e., in samples MA 233-234, ME 227-229, and AGO5 20-23, as can be seen also in the dashed blocks in Figure S5), from both $\mathrm{DH}$ and Coulter counter data. These events are marked by a very high particle concentration, especially at small diameters $\lesssim 1.5 / 2 \mu \mathrm{m}$, as revealed by Coulter Counter measurements. Moreover, we observe a large number of aggregates resulting from the increased probability for two particles to collide and stick together. We do not have access to this size range due to the size limit of the holographic instrument presented here; however, we still notice a particle concentration anomaly in the larger size range. We show in Figure 11 the results sorting the sites by the impact of wind erosion, increasing from left to right. To exclude possibly contaminated samples and anomalies, here we do not include samples containing volcanic (a)

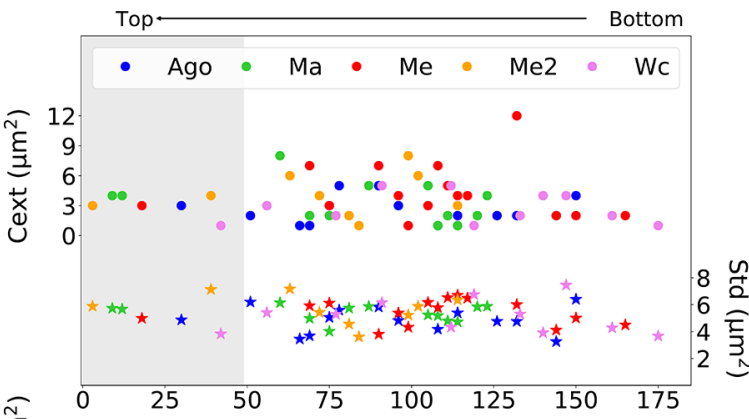

(b)

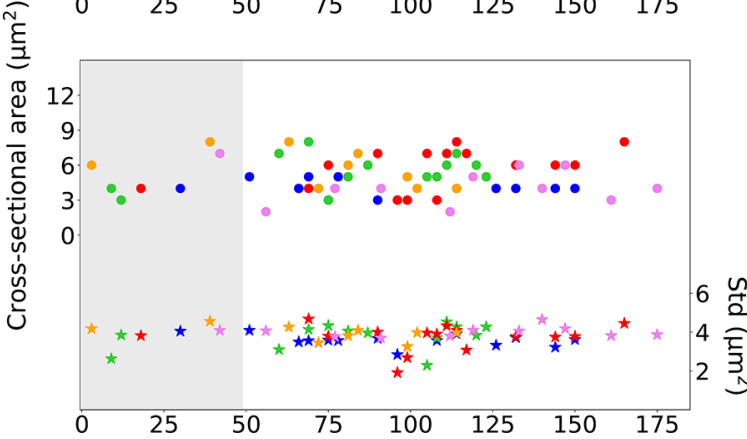

(c)

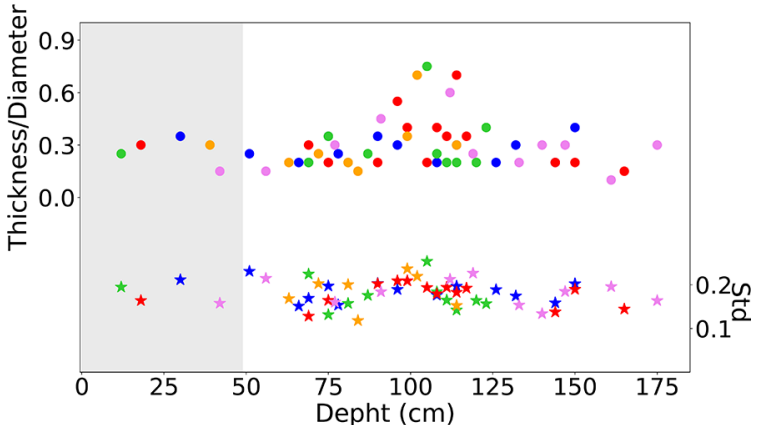

Figure 10. Summary of the results reported as a function of sample depth in $\mathrm{cm}$. (a) $C_{\text {ext }}$ (b) $c s a$, and (c) $t d r$ distribution of each sample (circles, left $y$-axis) and standard deviation (stars, right $y$-axis). Sites are color-coded: blue for AGO, green for MA, red for ME, orange for ME2 (another sample site of ME2), and violet for WC. The gray regions highlight samples close to the surface.

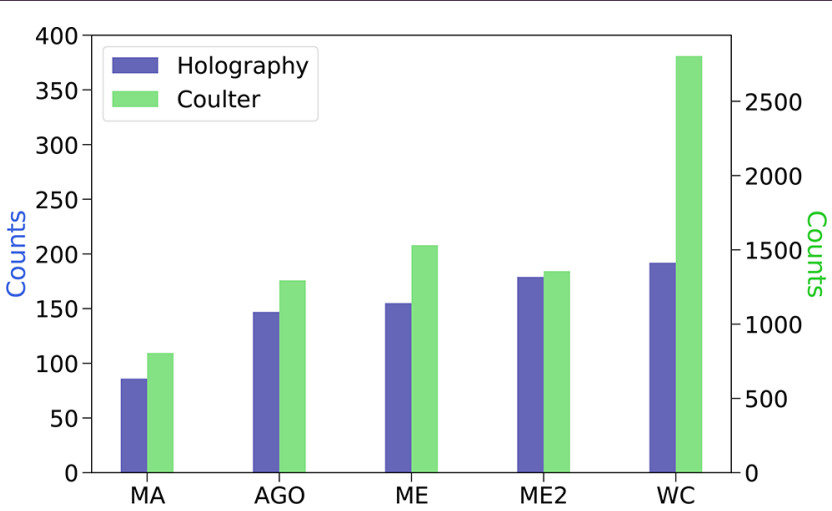

Figure 11. Comparison of the average particle counts for the five sites, excluding volcanic and superficial regions. The left $y$-axis refers to the $\mathrm{DH}$ measurements, whereas the $y$-axis on the right refers to Coulter Counter measurements on the same size range. The absolute counts differ due to the different sensitivities of the two instruments.

events nor samples close to the surface. We point out that comparing Coulter Counter and $\mathrm{DH}$ results for particle concentration and size distribution is not straightforward, as 
the physical quantities measured by these two methods are different. Here, we set an approximate threshold in size to compare the two results, from 1 to $20 \mu \mathrm{m}^{2}$, but this is intrinsically blurred by the fact that the Coulter Counter gives a volume-equivalent diameter for each particle, whereas holography gives a cross-sectional area. The relationship between these two quantities is not trivial, as it clearly follows from Figure 8. This is more evident in the WC samples, which have $\sim 3$ times the particle counts as the other sites. WC site is especially subject to sublimation by the wind while not exhibiting appreciable erosion, which produces crusts and alters the dust content, enhancing postdepositional aggregation. Anyway, we notice a distinct rise in dust concentration due to snow sublimation caused by the wind, in agreement with Coulter Counter measurements.

\section{CONCLUSIONS}

With the DH method proposed here, we are able to analyze size-polydisperse micrometric mineral dust particles in liquid suspensions and simultaneously provide information about the morphological and optical properties of each measured particle.

The instrument setup resembles a flow imaging microscope and the acquisition process is at least as fast. From an imaging standpoint, the resolution of the images reconstructed from the holograms is intrinsically lower than those from an equivalent optical microscope; on the other hand, the holograms contain usable information about the particle's optical properties. Particle size and shape can be easily identified, and image quality can be enhanced computationally.

The technique shows results that are compatible with Coulter Counter measurements; the setup works at low flow rates $(\sim 2 \mathrm{~mL} / \mathrm{min})$ and can be implemented in continuous flow analysis systems typically used in ice core characterization. In its current design, the instrument is able to effectively detect particles whose extinction cross section roughly above $C_{\text {ext }} \gtrsim$ $0.3 \mu \mathrm{m}^{2}$. In addition to the extinction cross section, we retrieve the particle shape, projected on a plane orthogonal to the optical axis.

Our analysis reveals that the majority of the particles in the samples deviate from the isometric shape, with a prevalence of oblate particles with a principal thickness to diameter ratio ranging between 0.15 and 0.4 . We find a remarkable variability in the extinction cross section of the particles depending on their thickness, for any given size. In the samples, no trace of highly absorbing particles was found. Experimental data indicates that the extinction cross section of isometric particles is up to 3 times that of plate-shaped particles. Indeed, experimental data highlight the importance of multiparametric characterization in order to obtain reliable particle sizing based on optical methods (as in optical particle counters): the size distribution is strongly model-dependent and varies significantly with the aspect ratio. Depending on its size, dust interaction with shortwave and longwave radiation and its impact on radiative transfer can range from a cooling to a warming effect. ${ }^{42}$ Similarly, accounting for dust asphericity leads to significant differences in the aerosol optical depth (compared to spheres). Deriving information about particle shapes is critical to determine the intrinsic single-scattering properties needed for state-of-the-art radiative transfer models. $^{22,43,44}$

\section{ASSOCIATED CONTENT}

\section{(s) Supporting Information}

The Supporting Information is available free of charge at https://pubs.acs.org/doi/10.1021/acsearthspacechem.1c00224.

Additional details on the techniques: validation procedure of the instrument with calibrated samples of known shape, some further insights on the determination of the optical parameters, and a more detailed comparison with the results of a state-of-art instrument, the Coulter Counter (PDF)

\section{AUTHOR INFORMATION}

\section{Corresponding Author}

Claudia Ravasio - Department of Physics, University of Milan, I-20133 Milan, Italy; ㅇo orcid.org/0000-0001-5986-1081; Email: claudia.ravasio@unimi.it

\section{Authors}

Llorenç Cremonesi - Department of Physics, University of Milan, I-20133 Milan, Italy

Claudio Artoni - Department of Earth and Environmental Sciences, University of Milano-Bicocca, I-20126 Milan, Italy; $\mathrm{Ca}$ ' Foscari University of Venice, Department of

Environmental Sciences, Informatics and Statistics, I-30172 Venice, Italy

Barbara Delmonte - Department of Earth and Environmental Sciences, University of Milano-Bicocca, I-20126 Milan, Italy

Valter Maggi - Department of Earth and Environmental Sciences, University of Milano-Bicocca, I-20126 Milan, Italy; National Institute of Nuclear Physics, Milano-Bicocca section, I-20126 Milan, Italy

Marco A. C. Potenza - Department of Physics and CIMAINA, University of Milan, I-20133 Milan, Italy; (1) orcid.org/0000-0002-9379-6540

Complete contact information is available at:

https://pubs.acs.org/10.1021/acsearthspacechem.1c00224

\section{Author Contributions}

C.R. assembled the instrument, performed the measurements, analyzed data, and wrote the manuscript in close collaboration with L.C.; C.A. and B.D. performed the Coulter Counter measurements and interpreted data; M.A.C.P., B.D., and V.M. contributed to the general overview of the work and gave support to the data analysis; all authors contributed to writing and editing the manuscript.

\section{Notes}

The authors declare no competing financial interest.

\section{ACKNOWLEDGMENTS}

The authors thank the ANR EAIIST project, grant ANR-16CE01-0011-01 of the French Agence Nationale de la Recherche, the BNP-Paribas foundation and its Climate Initiative Program, the Institut Polaire Français IPEV, the LabEx OSUG@2020 ("Investissements d'avenir" - ANR10 LABX56), the technical support from the F2G (French National platform for Coring and Drilling supported by INSU, the MIUR (Ministry of Education, University and Research) - PNRA (National Antarctic Research Program) through the EAIIST PNRA16 00049-B project, Australian Antarctic Science project number AAS 4537, the AAD, and the Institute of Marine and Antarctic Studies (IMAS). The authors 
thank Andrea Spolaor, Alexis Leluc, Anthony Vende, Quentin Celle, and Nicolas Rambauts for their technical support during the field traverse and the sampling of the snowpits. Laboratory activities were partially funded by the Italian Regional Affair Ministry.

\section{REFERENCES}

(1) Stocker, T. F.; Qin, D.; Plattner, G.-K.; Tignor, M.; Allen, S. K.; Boschung, J.; Nauels, A.; Xia, Y.; Bex, V.; Midgley, P. M.; et al. Climate change 2013: The physical science basis. Contribution of working group I to the fifth assessment report of the intergovernmental panel on climate change 2013, 1535.

(2) Carslaw, K. S.; Gordon, H.; Hamilton, D. S.; Johnson, J. S.; Regayre, L. A.; Yoshioka, M.; Pringle, K. J. Aerosols in the preindustrial atmosphere. Current Climate Change Reports 2017, 3, 1-15. (3) Choobari, O. A.; Zawar-Reza, P.; Sturman, A. The global distribution of mineral dust and its impacts on the climate system: A review. Atmos. Res. 2014, 138, 152-165.

(4) Massabò, D.; Caponi, L.; Bernardoni, V.; Bove, M.; Brotto, P.; Calzolai, G.; Cassola, F.; Chiari, M.; Fedi, M.; Fermo, P.; et al. Multiwavelength optical determination of black and brown carbon in atmospheric aerosols. Atmos. Environ. 2015, 108, 1-12.

(5) Claquin, T.; Schulz, M.; Balkanski, Y.; Boucher, O. Uncertainties in assessing radiative forcing by mineral dust. Tellus, Ser. B 1998, 50, 491-505.

(6) Kulkarni, P.; Baron, P. A.; Willeke, K. Aerosol measurement: principles, techniques, and applications; John Wiley \& Sons, 2011.

(7) Kärcher, B.; Möhler, O.; DeMott, P. J.; Pechtl, S.; Yu, F. Insights into the role of soot aerosols in cirrus cloud formation. Atmospheric Chemistry and Physics Discussions 2007, 7, 7843-7905.

(8) Randall, D. A.; Wood, R. A.; Bony, S.; Colman, R.; Fichefet, T.; Fyfe, J.; Kattsov, V.; Pitman, A.; Shukla, J.; Srinivasan, J. et al. Climate change 2007: The physical science basis. Contribution of Working Group I to the Fourth Assessment Report of the IPCC (FAR); Cambridge University Press, 2007; pp 589-662.

(9) Tegen, I.; Fung, I. Contribution to the atmospheric mineral aerosol load from land surface modification. J. Geophys. Res. 1995, 100, 18707-18726.

(10) Prospero, J. M.; Ginoux, P.; Torres, O.; Nicholson, S. E.; Gill, T. E. Environmental characterization of global sources of atmospheric soil dust identified with the Nimbus 7 Total Ozone Mapping Spectrometer (TOMS) absorbing aerosol product. Rev. Geophys. 2002, 40, 2-1.

(11) Kok, J. F.; Parteli, E. J.; Michaels, T. I.; Karam, D. B. The physics of wind-blown sand and dust. Rep. Prog. Phys. 2012, 75, 106901.

(12) Di Mauro, B. D.; Garzonio, R.; Rossini, M.; Filippa, G.; Pogliotti, P.; Galvagno, M.; di Cella, U. M.; Migliavacca, M.; Baccolo, G.; Clemenza, M.; et al. Saharan dust events in the European Alps: role on snowmelt and geochemical characterization. Cryosphere 2019, $13,1147-1165$.

(13) Ruth, U.; Barbante, C.; Bigler, M.; Delmonte, B.; Fischer, H.; Gabrielli, P.; Gaspari, V.; Kaufmann, P.; Lambert, F.; Maggi, V.; et al. Proxies and measurement techniques for mineral dust in Antarctic ice cores. Environ. Sci. Technol. 2008, 42, 5675-5681.

(14) Petit, J.-R.; Delmonte, B. A model for large glacial-interglacial climate-induced changes in dust and sea salt concentrations in deep ice cores (central Antarctica): palaeoclimatic implications and prospects for refining ice core chronologies. Tellus, Ser. B 2009, 61, $768-790$.

(15) Delmonte, B.; Paleari, C. I.; Andò, S.; Garzanti, E.; Andersson, P. S.; Petit, J. R.; Crosta, X.; Narcisi, B.; Baroni, C.; Salvatore, M. C.; et al. Causes of dust size variability in central East Antarctica (Dome B): Atmospheric transport from expanded South American sources during Marine Isotope Stage 2. Quat. Sci. Rev. 2017, 168, 55-68.

(16) Kahnert, M.; Nousiainen, T.; Veihelmann, B. Spherical and spheroidal model particles as an error source in aerosol climate forcing and radiance computations: A case study for feldspar aerosols. J. Geophys. Res. 2005, 110, 1 DOI: 10.1029/2004JD005558.

(17) Kahnert, M.; Nousiainen, T.; Räisänen, P. Mie simulations as an error source in mineral aerosol radiative forcing calculations. Q. J. R. Meteorol. Soc. 2007, 133, 299-307.

(18) Potenza, M.; Cremonesi, L.; Delmonte, B.; Sanvito, T.; Paroli, B.; Pullia, A.; Baccolo, G.; Maggi, V. Single-particle extinction and scattering method allows for detection and characterization of aggregates of Aeolian dust grains in ice cores. ACS Earth and Space Chemistry 2017, 1, 261-269.

(19) Mehri, T.; Kemppinen, O.; David, G.; Lindqvist, H.; Tyynelä, J.; Nousiainen, T.; Rairoux, P.; Miffre, A. Investigating the size, shape and surface roughness dependence of polarization lidars with lightscattering computations on real mineral dust particles: Application to dust particles' external mixtures and dust mass concentration retrievals. Atmos. Res. 2018, 203, 44-61.

(20) Liu, L.; Mishchenko, M. I. Scattering and radiative properties of morphologically complex carbonaceous aerosols: A systematic modeling study. Remote Sensing 2018, 10, 1634.

(21) Dubovik, O.; Sinyuk, A.; Lapyonok, T.; Holben, B. N.; Mishchenko, M.; Yang, P.; Eck, T. F.; Volten, H.; Munoz, O.; Veihelmann, B. Application of spheroid models to account for aerosol particle nonsphericity in remote sensing of desert dust. J. Geophys. Res. 2006, 111, 1 DOI: 10.1029/2005JD006619.

(22) Potenza, M.; Albani, S.; Delmonte, B.; Villa, S.; Sanvito, T.; Paroli, B.; Pullia, A.; Baccolo, G.; Mahowald, N.; Maggi, V. Shape and size constraints on dust optical properties from the Dome $\mathrm{C}$ ice core, Antarctica. Sci. Rep. 2016, 6, 1-9.

(23) Huang, Y.; Kok, J. F.; Kandler, K.; Lindqvist, H.; Nousiainen, T.; Sakai, T.; Adebiyi, A.; Jokinen, O. Climate models and remote sensing retrievals neglect substantial desert dust asphericity. Geophys. Res. Lett. 2020, 47, e2019GL086592.

(24) Philips, L. A.; Ruffner, D. B.; Cheong, F. C.; Blusewicz, J. M.; Kasimbeg, P.; Waisi, B.; McCutcheon, J. R.; Grier, D. G. Holographic characterization of contaminants in water: Differentiation of suspended particles in heterogeneous dispersions. Water Res. 2017, 122, 431-439.

(25) Nadeau, J.; Cho, Y. B.; El-Kholy, M.; Bedrossian, M.; Rider, S.; Lindensmith, C.; Wallace, J. K. Holographic microscopy for 3D tracking of bacteria. Proc. SPIE 2016, 97182B.

(26) Kasimbeg, P. N.; Cheong, F. C.; Ruffner, D. B.; Blusewicz, J. M.; Philips, L. A. Holographic characterization of protein aggregates in the presence of silicone oil and surfactants. J. Pharm. Sci. 2019, 108, $155-161$.

(27) Kemppinen, O.; Laning, J. C.; Mersmann, R. D.; Videen, G.; Berg, M. J. Imaging atmospheric aerosol particles from a UAV with digital holography. Sci. Rep. 2020, 10, 1-12.

(28) Gabor, D. A New Microscopic Principle. Nature 1948, 161, 777.

(29) Kaufmann, P. R.; Federer, U.; Hutterli, M. A.; Bigler, M.; Schüpbach, S.; Ruth, U.; Schmitt, J.; Stocker, T. F. An improved continuous flow analysis system for high-resolution field measurements on ice cores. Environ. Sci. Technol. 2008, 42, 8044-8050.

(30) Bigler, M.; Svensson, A.; Kettner, E.; Vallelonga, P.; Nielsen, M. E.; Steffensen, J. P. Optimization of high-resolution continuous flow analysis for transient climate signals in ice cores. Environ. Sci. Technol. 2011, 45, 4483-4489.

(31) Van de Hulst, H. Light scattering by small particles (Courier Corporation); Dover Publications, Inc.: New York, NY, USA, 1957.

(32) Bohren, C. F.; Huffman, D. R. Absorption and scattering of light by small particles; John Wiley \& Sons, 2008.

(33) Potenza, M.; Sabareesh, K.; Carpineti, M.; Alaimo, M.; Giglio, M. How to measure the optical thickness of scattering particles from the phase delay of scattered waves: application to turbid samples. Phys. Rev. Lett. 2010, 105, 193901.

(34) Berg, M. J.; Subedi, N. R.; Anderson, P. A.; Fowler, N. B. Using holography to measure extinction. Opt. Lett. 2014, 39, 3993-3996. 
(35) Berg, M. J.; Subedi, N. R.; Anderson, P. A. Measuring extinction with digital holography: nonspherical particles and experimental validation. Opt. Lett. 2017, 42, 1011-1014.

(36) Krishnatreya, B. J.; Grier, D. G. Fast feature identification for holographic tracking: The orientation alignment transform. Opt. Express 2014, 22, 12773-12778.

(37) Schnars, U.; Falldorf, C.; Watson, J.; Jüptner, W. Digital Holography and Wavefront Sensing; Springer, 2015; pp 39-68.

(38) Latychevskaia, T.; Fink, H.-W. Practical algorithms for simulation and reconstruction of digital in-line holograms. Appl. Opt. 2015, 54, 2424-2434.

(39) Simonsen, M. F.; Cremonesi, L.; Baccolo, G.; Bosch, S.; Delmonte, B.; Erhardt, T.; Kjær, H. A.; Potenza, M.; Svensson, A.; Vallelonga, P. Particle shape accounts for instrumental discrepancy in ice core dust size distributions. Climate of the Past 2018, 14, 601-608.

(40) Paleari, C. I.; Delmonte, B.; Andò, S.; Garzanti, E.; Petit, J. R.; Maggi, V. Aeolian dust provenance in central East Antarctica during the Holocene: Environmental constraints from single-grain Raman spectroscopy. Geophys. Res. Lett. 2019, 46, 9968-9979.

(41) Yurkin, M. A.; Hoekstra, A. G. The discrete-dipoleapproximation code ADDA: capabilities and known limitations. J. Quant. Spectrosc. Radiat. Transfer 2011, 112, 2234-2247.

(42) Huang, Y.; Adebiyi, A. A.; Formenti, P.; Kok, J. F. Linking the different diameter types of aspherical desert dust indicates that models underestimate coarse dust emission. Geophys. Res. Lett. 2021, 48, e2020GL092054.

(43) Meng, Z.; Yang, P.; Kattawar, G. W.; Bi, L.; Liou, K.; Laszlo, I. Single-scattering properties of tri-axial ellipsoidal mineral dust aerosols: A database for application to radiative transfer calculations. J. Aerosol Sci. 2010, 41, 501-512.

(44) Kok, J. F.; Ridley, D. A.; Zhou, Q.; Miller, R. L.; Zhao, C.; Heald, C. L.; Ward, D. S.; Albani, S.; Haustein, K. Smaller desert dust cooling effect estimated from analysis of dust size and abundance. Nat. Geosci. 2017, 10, 274-278. 